\title{
PocketInjector: Smart Phone based Insulin Delivery System
}

\author{
Soumyakant Sahoo \\ NBNSSOE,Pune,India \\ Department of Information \\ Technology
}

\author{
Pooja Doshi \\ NBNSSOE,Pune,India \\ Department of Information \\ Technology
}

\author{
Rahul Samant \\ Assistant Professor \\ NBNSSOE (I.T. Dept) \\ Pune,India
}

\begin{abstract}
In developing countries like India, providing healthcare services to the rural population having low income has been a challenge due to scarcity of medical practitioners. The distribution of available skilled healthcare resources is uneven with large concentration in urban areas and villages. This requires venturing into ways to increase the reach of healthcare services catering to low income rural population. One of such emerging ways of providing healthcare services is through smart phone applications. As per facts stated by Internet and mobile association of India in the 2015, India will reach 236 million smart phone users by 2016 . One of the killing widespread diseases which require urgent attention is diabetes mellitus. India is called the diabetes capital of the world where according to a survey done in 2015. Contrary to popular belief, diabetes mellitus affects more people in rural areas (34 million) than affluent urban cities (28 million). Till date smart phone based apps such as Diabetes Logbook were used in diabetes healthcare as just reminders for insulin intake through syringe. In the implementation of smart phone hardware extension called PockeInjector, along with function such as notification for insulin intake through syringe, authors propose a hardware attached to smart phone that can facilitate injecting of insulin into the patients body added with function of notifying family members or family doctors about the date, time and location of patient while taking the insulin dosage. The functioning of injecting insulin through syringe is carried out by the hardware that is controlled by Android ${ }^{\circledR}$ smart phone based application that also sends notification about the intake process and manages the reminders for taking insulin dosage.
\end{abstract}

This novel approach combines the traditional procedure with a smart phone application.

\section{General Terms}

Healthcare automation, smart phone hardware extension, arduino-insulin delivery system.

\section{Keywords}

PocketInjector, Smart Phone Based Insulin Delivery System, Android $\AA$ based Diabetic Application, Android ${ }^{\circledR}$ based healthcare automation.

\section{INTRODUCTION}

Developing countries like India and its neighbours have large percentage of rural population with low income. People in rural areas suffer from life threatening diseases and infections such as Human Immunodeficiency Virus (HIV), Diabetes mellitus and Tuberculosis. One of the main reasons for this is lack of education and resources for awareness amongst people. It is a challenge to provide health care services to the rural population due to scarcity of medical practitioners. Skilled health care resources are easily available in urban areas as against in rural areas.

According to the survey [11], diabetes mellitus is one such potentially dangerous disease in India which requires proper medical attention and care. India is called the diabetes capital of world according to the survey done by [11] in 2015 . Majority of people in rural areas are suffering from diabetes mellitus (34 million ) as compared to people in urban areas (28 million) [11]. Diabetes like diseases are silent killers. People tend to ignore such diseases till it reaches fatal state. Due to late diagnosis and ignorance, diabetes mellitus can be a big risk for long term health. There are many side effects of leaving diabetes mellitus untreated. Some of the side effects can be vision loss, vascular dementia (vascular damage), ketoacidosis, cardiovascular and nerve damage [12].

Research suggests that linking health care with other services such as shelter and food might help improve the continuity of care [10]. KhushiBaby is one such attempt to provide health care services for babies. It seeks to improve vaccination rates with a necklace that digitizes data at the point of care [10].As per the facts stated by Internet and Mobile association of India in 2015, India will be home for 236 million smart phone users by 2016 . Fortunately, the spread of smart phone use is gaining momentum in India [14].

Many smart phone applications are in a stage of development to treat diabetes mellitus effectively. Diabetes Logbook is one such smart phone based application which helped diabetic patients to take insulin dosages in a form of an injection with periodical reminders [13]. According to medical practitioners, reminders alone are not effective means for treatment [15]. Authors propose to combine reminders and dosage intake procedure to make it more effective.

\section{LITERATURE SURVEY}

In the paper- Doctor's Orders: Healthcare Apps and self monitoring (2014), Thomas Jespen discussed on the needs for healthcare applications which will reduce the burden of doctors and can help people improve their fitness levels and reduce risky behaviors. However the challenges in developing such healthcare application needs to addressed thoroughly.

Proposing on similar lines, D. Feng et. al. (2015) proposed a platform, Guest Editorial Telehealth Systems and Applications, for developing Telehealth services and applications for efficient solutions to health related problems. Challenges for developing such Telehealth services and applications as mentioned above can be really challenging in terms of mobility of devices delivering the health services and acceptability by individual users as mentioned in [6].

Addressing the issue of user acceptibility of Telehealth services [9], David Moloney and Oscar Deniz Suarez, in the paper- A vision for the Future (2015), have described the 
technologies that can be used to enhance user's experiences for wide range of applications and the research seems bright and appealing.

Telehealth services and applications can be more effective and scores more on enhanced user experience [8] if delivered via wearable computing devices. In the year 2014, Thad Starner contributed to the research on Wearable Computing devices and explained the challenges in developing the devices. These challenges as mentioned in [6] needs to be met with solutions for taking forward Telehealth services and applications[9] to enhance user's experience for wide range of applications [8].

In the paper- Emerging Technologies for e-healthcare, published in the year 2012, Xuemin (Sherman) Shen described a system with 3 domains: body area, communication and networking, and services. Body area consists of sensors that continuously monitor user's physiological conditions and the server- receives, records and monitors user's health related information. Using sensors and servers continuously might face the challenge of energy efficiency and reliable information collection.

Adding to the domain of e-healthcare Technologies [2] and Telehealth applications [Telehealth], Andrew Clarke and Robert Steele, in the year 2014, described a smart phone based application System for public health data collection and intervention. The system consists of a server that records and monitors the data of public health without the need for identifying the details of an individual participant to ever leave his/her mobile device. In such systems, while identifying user and providing privacy, a common set of rules is needed to be set as a compromise between individual privacy and public health application requirements.

Julie Barnett et. al. (2014) described myPace: An Integrative Health Platform for supporting Weight Loss and maintenance behaviors, a system that is deployed through smart phone and personal computer. Findings show that system requires development in order to be fully acceptable within diabetic practice. Moreover the need of personal computer makes it less portable.

In the paper- Youth Street Connect- helping Homeless Young Women, Silvia Figueria et. al. (2014) have described a tool formed by 2 mobile applications which will connect young homeless young women to local healthcare providers. In this tool healthcare providers are needed to monitor and provide services for their individual needs. Also healthcare providers will be inefficient if homeless youth are unaware of services available.

Peter G. Jacobs et. al. (2011) have described a Fully Automated Insulin Delivery System. The system consists of sensors which monitors user's blood sugar level and accordingly pumps insulin or glucagon in the user's body. A custom software application that controls the sensor acquisition and insulin and glucagon delivery requires sensors for constantly sensing blood sugar level. To run this custom software application, a laptop is also needed. These requirements might become a major drawback of the system.

\section{PROPOSED METHODOLOGY}

Authors propose a smart phone based insulin delivery system called PocketInjector. It is a smart phone hardware extension comprising of two basic units, 1: Software in the form of PocketInjector, an Android® application and 2: Hardware in form of Arduino UNO or Freeduino, an Arduino variant.

The PocketInjector - an Android ${ }^{\circledR}$ application is based on
API level 14, supporting smart phones with the mobile operating system Android® 4.0 (Ice-cream Sandwich).

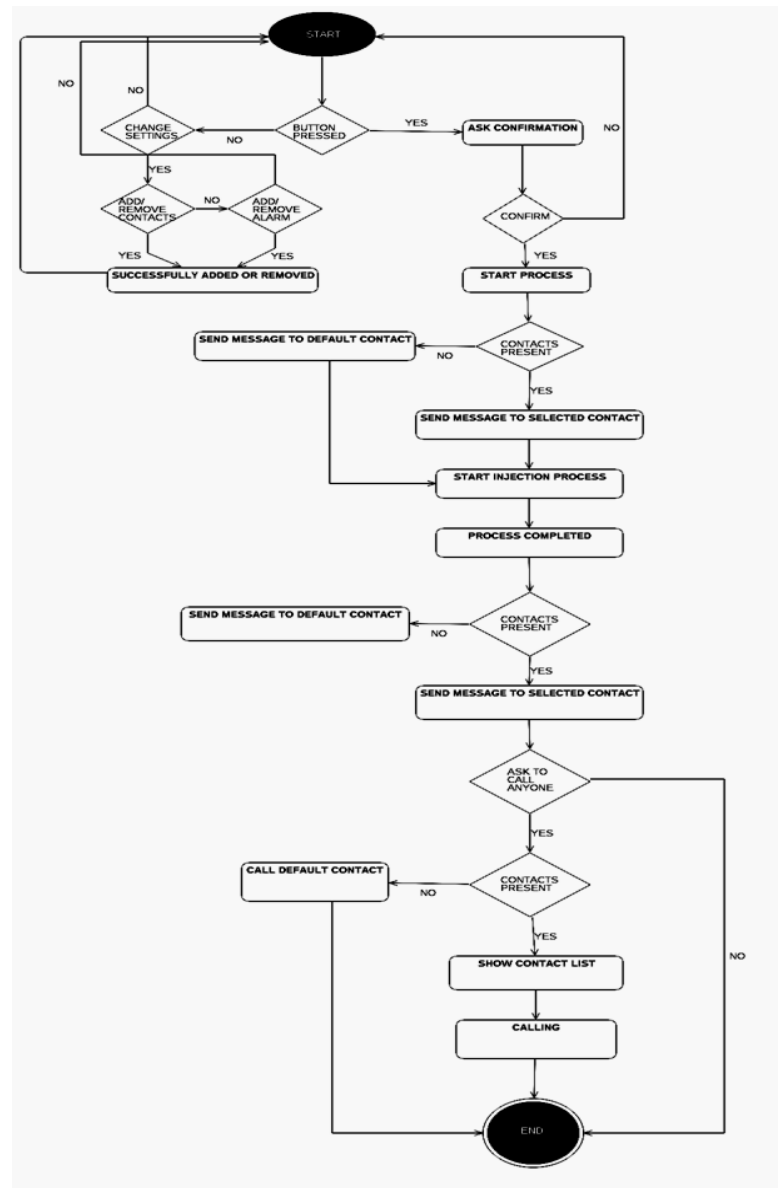

Fig 1: System Flowchart for PocketInjector

PocketInjector provides functionality of sending notification in the form of text messages to the user configured emergency contacts and default contact on starting and completion of insulin intake procedure. It also provides the functionality of calling any individual person from emergency contact list or default contact. User can configure PocketInjector with emergency contacts, default contact, and default messages easily. Proposed application supports emergency mode operation over the locked screen with full brightness and ringer volume. Emergency mode can be triggered by attaching the hardware unit to the USB (Universal Serial Bus) charging slot of the smart phone. Major functionality of PocketInjector includes triggering the attached hardware extension to inject insulin into intended body part of the human user under controlled environment and pulling out the needle after completion of the procedure.

The hardware unit comprises of an Arduino UNO or Freeduino connected to smart phone through type-2 USB serial interfacing. Arduino is one of the two popular microcontroller used in robotics industry for various applications, other being Raspberry-pi which is a complete computer on a chip. Since PocketInjector has a defined and specific working, authors have opted for Arduino that can be programmed for specific working mechanisms in contrary to Raspberry-pi which can do varied tasks like a computer. 


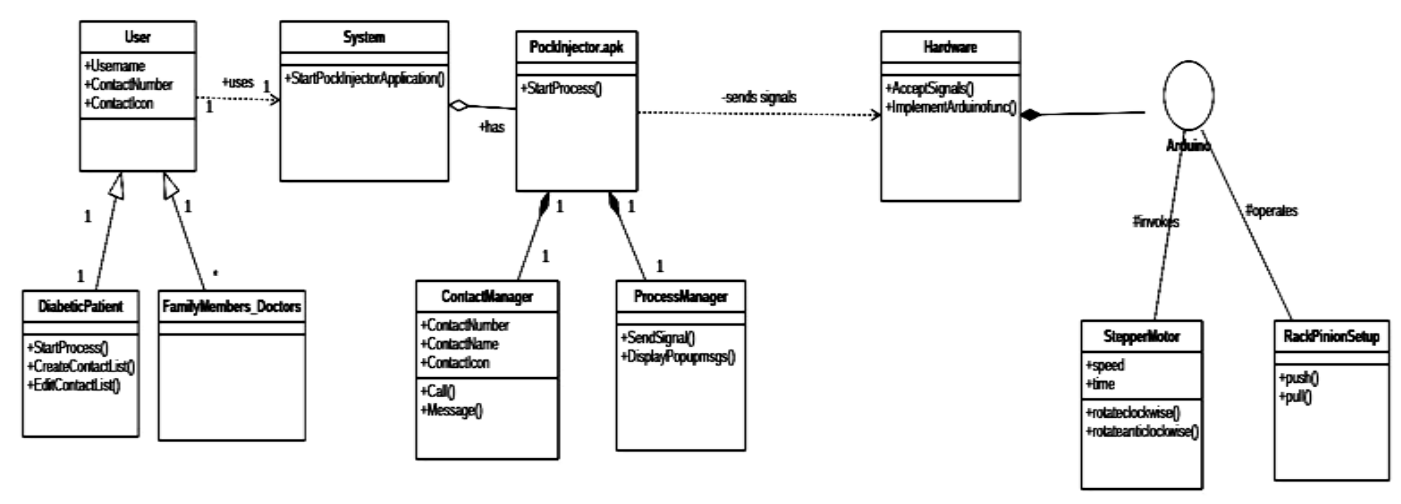

\section{Fig 2: Class Diagram of PocketInjector}

Interfacing requires connecting the hardware unit with smart phone via USB On-the-go (OTG) cable that allows smart phone to act as host for the hardware unit. The hardware unit draws power from the smart phone for its functioning, making it convenient to carry around. The hardware unit is mounted with a $12 \mathrm{~V}$ DC stepper motor providing torque of $2 \mathrm{~kg} \mathrm{~cm}$ and a $12 \mathrm{~V}$ battery that serves the purpose of pushing and pulling the insulin injection on trigger from the PocketInjector application.

Proposed methodology makes the insulin intake procedure smart and safer by converting it into smart phone hardware extension that controls the entire insulin injection process and simultaneously notifies user specified contacts about the procedure with an additional possibility of immediate help in cases of emergency.

\section{DISCUSSION}

PocketInjector system proposed here, can be considered as advancement on the system proposed in [1]. In contrast to architecture proposed in [1], architecture being proposed here is more flexible and portable. PocketInjector application can be installed on smart phones instead of large bulky PCs/Laptops. The small hardware unit is easy to carry around and can be attached to smart phones using a USB OTG cable. Proposed architecture is composed of -:

\section{PocketInjector Android®application}

\section{Arduino UNO/Freeduino micro-controller}

\section{Insulin pen}

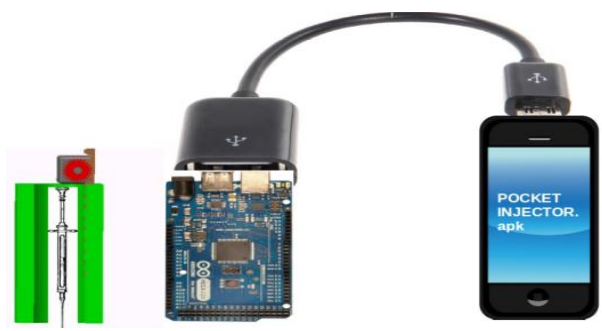

Fig 3: High Level architecture of the system

Use Android ${ }^{\circledR}$ smart phone for delivering insulin through insulin pen is more efficient and portable as compared to [1], where a laptop or PC is always required for monitoring and delivery of insulin. Arduino UNO or Freeduino is widely used microcontroller in various robotics applications that can be used to operate the insulin pen through a stepper motor. Stepper motor mounted on Arduino Uno/ Freeduino gives the freedom of controlling the motor operation programmatically and hence making the entire procedure more user-friendly. Use of Android ${ }^{\circledR}$ smart phone makes the system more appealing and acceptable by large number of users when compared to the number of users that will find using PC and sensor based insulin delivery system easy to understand and use. This advantage of PocketInjector is a major advantage considering the fact that number of people suffering from diabetes and requiring insulin is large in India.

\section{RESULTS}

PocketInjector is a promising system in terms of automating the insulin delivery procedure and making it smart. Components of PocketInjector are widely used by people, whether it be Android $\AA$ smart phones or arduino UNO/ freeduino, which is widely used in industry for robotics application. Authors have brought these components together to develop PocketInjector, which is a smart, self operated and portable way of insulin delivery. The PocketInjector application is easy to use and provides one-click delivery of insulin with the help of interfaced hardware. Considering the components used, PocketInjector can be a promising candidate for mass production for healthcare industry.

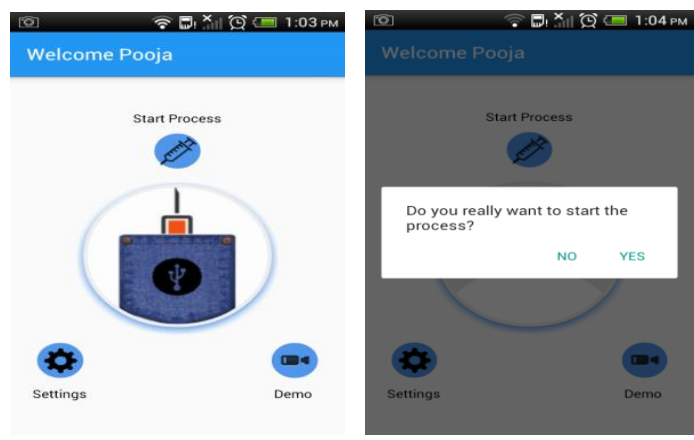

Fig 4: Snapshot of starting the system

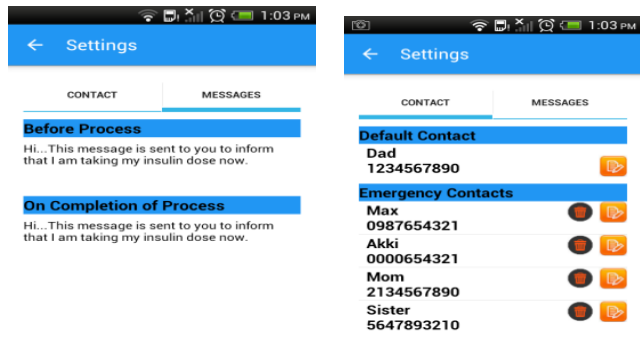

EDIT MESSAGE

Fig 5: Snapshot of Message and Contact Settings 


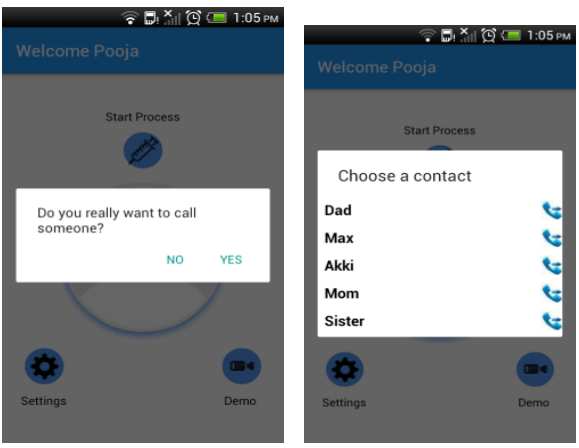

Fig 6: Snapshot for making a call

With more brains coming together for mass production of PocketInjector, it cannot be denied that the system can be made more affordable for use.

\section{CONCLUSION AND FUTURE SCOPE}

Authors have proposed PocketInjector insulin delivery system as a prototype that can be a potential system for making the insulin delivery procedure smart and portable. Further research in developing similar healthcare wearables or smart phone hardware extensions can go a long way in minimizing the need of face to face interaction of patients and doctors although ensuring that doctors are well informed about there patients condition. PocketInjector is a system exploiting the power of available technology around us to enhance the quality of individual health.

Future scope for PocketInjector can be making it a generic system that can be used with different smart phone, increasing the application functionalities by adding video conferencing with doctors and dispense of pills through the system.

\section{ACKNOWLEDGMENTS}

The authors would like to thank Persistent Systems Ltd. for sponsoring the project and Mr. Milind Deshpande, Mr. Milind Suryawanshi for guiding and mentoring the project on behalf of Persistent Systems Ltd.

Our sincere thanks to Prof. Shivprasad Patil (Head of the Department, I.T, NBN Sinhgad School of Engineering, Ambegaon, Pune-41) for his support and guidance throughout the project.

\section{REFERENCES}

[1] Peter G. Jacobs, Joseph El Youssef, Jessica R. Castle, Julia M. Engle, Deborah L. Branigan, Phillip Johnson, Ryan Massoud, Apurv Kamath, W. Kenneth Ward, "Development of a fully automated closed loop artificial pancreas control system with dual pump delivery of insulin and glucagon", Engineering in Medicine and Biology Society, EMBC , 2011 Annual International Conference of the IEEE, DOI: 10.1109/IEMBS.2011.6090127, PubMed ID: 22254332, On page(s): 397-400, August 30 2011- September 32011

[2] Shermen Shen, "Emerging Technologies for eHealthcare", IEEE Network vol:26 issue:5, DOI:10.1109/MNET.2012.6308066, On page(s): 2-3, September-October 2012
[3] Andrew Clarke, Robert Steele, "A Smart phone-Based System for Population-Scale Anonymized Public Health Data Collection and Intervention", System Sciences (HICSS), 2014 47th Hawaii International Conference on, DOI:10.1109/HICSS.2014.363, On page(s): 2908-2917, 6-9 January 2014

[4] Thad Starner, "How Wearables Worked their Way into the Mainstream", IEEE-Pervasive Computing vol:13 issue:4, DOI: 10.1109/MPRV.2014.66, On page(s):1015, October- December 2014

[5] Silivia Figueira, Kelsey Dedoshka, Katie Le, Kaitlin Kirasich, Deb Levine, "Youth StreetConnect - Helping homeless young women", Global Humanitarian Technology Conference (GHTC), 2014 IEEE, DOI:10.1109/GHTC.2014.6970348, On page(s):620-627, 10-13 October 2014

[6] Thomas Jespen, "Doctor's Orders: Healthcare Apps and self monitoring", IT Professional vol:16 issue:4, DOI:10.1109/MITP.2014.49, On page(s):48-49, JulyAugust 2014

[7] Julia Barnett, Michel Harricharan, Dave Fletcher, Becky Gilchrist, Jane Coughlan, "myPace: An Integrative Health Platform for Supporting Weight Loss and Maintenance Behaviors", IEEE Journal of Biomedical and Health Informatics vol:19 issue:1, DOI: 10.1109/JBHI.2014.2366832, On page(s):109-116, 25 November 2014

[8] David Moloney, Oscar Deniz Suarez, "A Vision for the Future [Soapbox]", IEEE Consumer Electronics Magazine, vol:4 issue:2 , DOI:10.1109/MCE.2015.2392956, On page(s):40-45, April 2015

[9] D. Feng, J. Kim, M. Khadra, D. L. Hudson, C. Roux, "Guest Editorial: Telehealth Systems and Applications", IEEE Journal of Biomedical and Health Informatics, vol:19 issue:1, DOI:10.1109/JBHI.2014.2380132, On page(s):40-45, January 2015

[10] Guest Writer, "5 mHealth Innovations Using Mobile Phone Extensions and Werables", www.ictworks.org, July 312015

[11] Sujatha, "Prevalance of Diabetes in India", www.mapsofindia.com, September 30, 2015, for statistics.

[12] https://www.betterhealth.vic.gov.au, August 25, 2015, for study of complications of untreated diabetes milletus

[13] https://play.google.com/store, mySugr GmbH, October 25,2015 , for study of existing application systems

[14] https://buisnessworld.in, September 21, 2015, for analysis of smart phone usage in India.

[15] http://www.ncbi.nlm.nih.gov, September 28, 2015, for suggestions about the improvements in the existing application systems 